\title{
A Survey of IUE Spectra of the Active Binary System UX Arietis
}

\author{
Fehmi Ekmekçi ${ }^{\mathrm{A}}$ \\ A Ankara University, Faculty of Science, Department of Astronomy and Space Sciences, \\ 06100 Tandoğan, Ankara, Turkey. Email: fekmekci@science.ankara.edu.tr
}

Received 2009 March 10, accepted 2009 August 27

\begin{abstract}
To investigate the ultraviolet (UV) activity of the bright, non-eclipsing, double-lined spectroscopic binary system UX Ari, IUE spectra (194 images) were taken from the IUE archive. The spectra, obtained during the period 1978-1996, show emission lines originating in the chromosphere and transition region. The long-wavelength low dispersion spectra were examined for ultraviolet excess by comparing the UV continuum level of UX Ari with the levels of $\kappa$ Cet and $\eta$ Cep in the spectral range 2100-3200 $\AA$. The individual MgII $h$ and $k$ emission-line fluxes of component stars show that the contributions to the activity of the system for G5 V and K0 IV are about $20 \%$ and $80 \%$, respectively. Apart from the flare event observed on 1979 Jan 1, there are some flux enhancements in the years 1987, 1991 and 1994 which may suggest existence of a periodicity of about 7-9 years. Examination of the ultraviolet excess in the system showed that there is some UV excess in UX Ari, which varies from $1 \%$ up to $24 \%$ with the exception of two images which showed no UV excess. The results revealed that there was an agreement on the source of emission regions which could be attributed to magnetic activity. The contribution of G5 V and K0 IV components to the MgII activity of the system suggests a need to take into consideration the spot distribution not only on the surface of the K0 IV but also on the surface of the G5 V component of UX Ari.
\end{abstract}

Keywords: binaries: spectroscopic — stars: activity — stars: chromospheres — stars: individual (UX Ari)

\section{Introduction}

UX Ari is a bright non-eclipsing double-lined spectroscopic binary $\left(P_{\text {orb }}=6.43791\right.$ days $)$ of spectral type G5 V+K0 IV (Carlos \& Popper 1971). The primary star, in this case the K0 IV component, shows chromospheric activity and is responsible for the majority of the activity shown by the system, as in most such RS Cvn systems. Some photometric and orbital characteristics of UX Ari are summarized in Table 1.

Huenemoerder, Buzasi \& Ramsey (1989) summarized the previous observations that had been done by numerous investigators, including photometric, spectroscopic, $\mathrm{X}$-ray, radio and ultraviolet features. They suggested that the excess absorption was due to the mass-transfer activity resulting from the Roche lobe over-flow of the K star and accretion onto $\mathrm{G}$ star by analyzing their $\mathrm{H} \alpha$ and $\mathrm{H} \beta$ observations obtained as fibre-optic, echelle, CCD spectra. Vogt \& Hatzes (1991) derived an accurate measurement of differential rotation with the opposite feature to that of the Sun using Doppler Imaging Technique. They also showed the spot distribution, which was quite complex, and the primary component, which had a large, stable polar spot.

Duemmler \& Aarum (2001) attempted to improve the orbital measurements of UX Ari by using the published radial velocities together with their high-accuracy data. They improved the set of orbital parameters and found that the $\gamma$ velocity of the system has a systematic variation with time. They concluded that UX Ari seemed to be a triple
Table 1. The characteristics of UX Ari

\begin{tabular}{lll}
\hline Parameter & & References \\
\hline$\alpha_{2000}$ & $03^{\mathrm{h}} 26^{\mathrm{m}} 35.36^{\mathrm{s}}$ & $\mathrm{a}$ \\
$\delta_{2000}$ & $+28^{\circ} 42^{\prime} 55.2^{\prime \prime}$ & $\mathrm{a}$ \\
Distance/pc & 50.23 & $\mathrm{a}$ \\
$P_{\text {orb }} /$ days & 6.43791 & $\mathrm{~b}$ \\
Spectral type & Hot: G5 V & $\mathrm{c}$ \\
& Cool: K0 IV & \\
Component masses $/ M_{\odot}$ & Hot: $\geq 0.93$ & $\mathrm{c}$ \\
& Cool: $\geq 0.71$ & \\
Component radii/ $R_{\odot}$ & Hot: 0.93 & $\mathrm{c}$ \\
& Cool: $>4.7$ & \\
$M_{\mathrm{v}}$ & 2.5 & $\mathrm{c}$ \\
$V$ & 6.38 & $\mathrm{c}$ \\
$B-V$ & 0.91 & $\mathrm{c}$ \\
$U-B$ & 0.48 & $\mathrm{c}$ \\
$i$ & $60^{\circ}$ & $\mathrm{c}$ \\
\hline
\end{tabular}

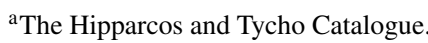

${ }^{\mathrm{b}}$ Carlos \& Poper (1971).

${ }^{\mathrm{c}}$ Strassmeier et al. (1993).

system. The excess emission/absorption in some chromospheric lines (see e.g. Montes et al. 1995a,b for $\mathrm{H} \alpha$ emission, and Huenemoerder et al. 1989 for $\mathrm{H} \alpha$ absorption) or the excess in continuum levels of various spectral ranges, if they exist, could provide evidence for the source 
of activity being dependent on rotation (related to magnetic activity), or an accretion stream from Roche-lobe overflow of the primary, or some process that occurs in hot circumstellar gas (see Rhombs \& Fix 1977), respectively. Therefore, the examination of the ultraviolet excess would also be advantageous.

Ekmekçi (1993) studied 65 IUE spectra observed in the 1978-1987 period (with IUESIPS reduction). It was shown that emission-line fluxes vary with the orbital phase and that the dependence of line flux on orbital phase was well correlated with the photometric light variation. This correlation might indicate more active chromospheric regions above the photospheric spot regions. Ekmekçi (1993) also measured fluxes of the individual IUESIPS emission lines of the component stars of UX Ari and calculated that the contributions to the activity of the system for G5 and K0 were about $1 / 4$ and 3/4 respectively. Another characteristic of the $I U E$ spectra (with IUESIPS reduction) was an absorption feature observed on the peak of the IUESIPS $k$ profiles of the K0 IV component, which was observed to shift together with the emission profile as the star revolved in its orbit (Ekmekçi 1993). Based on this absorption feature, it is suggested that the circumstellar matter around the K0 IV component may be responsible for this absorption.

Nichols \& Linsky (1996) summarized a new calibration together with new image processing techniques. They pointed out that the wavelength and absolute flux errors in the IUESIPS processing could be corrected by NEWSIPS progressing and that it would be better to re-analyze all the IUE spectra with NEWSIPS reduction. With this aim, in the present paper all 194 images of IUE-NEWSIPS spectra of UX Ari observed in 1978-1996 period have been analyzed to check the validity of the previous findings of Ekmekçi (1993). This paper shows that all the integrated emission-line fluxes of short-wavelength, low-dispersion spectra have a variation with time and orbital phase, but that the variation with time was not as clear as that with the orbital phase. Examination of the ultraviolet excess shows that some UV excess in UX Ari exists and varies from $1 \%$ up to $24 \%$ in time. Comparison of the $\mathrm{MgII}$ radial velocities with those of visible spectra (Carlos \& Popper 1971; Duemmler \& Aarum 2001) showed that the scattering in the UV data is likely to come from chromospheric activity caused by a magnetic dynamo that produces loops in active regions.

\section{IUE Data and Spectral Analysis}

The IUE spectra of UXAri were extracted from the NASA $I U E$ archive using IDL. All the spectra have undergone NEWSIPS reduction. The spectra consist of 22 LWP, 2 LWR and 86 SWP images in low dispersion, and 69 LWP, 12 LWR and 3 SWP images in high resolution. The log of IUE images is given in Table 2. The images studied by other authors in the past are denoted by asterisks in the 'Comment' column of Table 2. IUE obtained spectra at both low ( $6-\AA$ resolution) and high dispersion $(\lambda / \delta \lambda$ $\sim 10000$ ), with the short-wavelength prime (SWP, 1151$2000 \AA$ ), long-wavelength prime (LWP, 1850-3400 A), and long-wavelength redundant (LWR, 1850-3400 cameras (Nichols \& Linsky 1996).

The spectra show emission lines originating in the chromosphere and transition region. The flux in a given line was obtained by computing the area contained in the spectral region above the continuum or background levels near the wings of the line. The emission line fluxes were computed based on Gaussian profile-fitting procedures. The fitting procedures were made by means of the CURFIT program of Bevington (1969). Some results of the Gaussian fits are shown in Figure 1 for the lowdispersion image, SWP02375, and in Figure 6 for the high-resolution images, LWP14085 and LWP14130, in the range that includes the MgII $h$ and $k$ lines. The overall shapes of the line profiles can be reasonably well matched by 1 or 2 Gaussian components for low-dispersion SWP spectra and by 3 Gaussian components for $\operatorname{MgII} h$ and $k$ profiles in high-resolution images. In the case of Sirv or SiII lines there are two Gaussian components for the multiplets in the fitting procedure. The strong emission in these lines originates from the K0 IV star rather than G5 V star of UX Ari system. In the case of the NEWSIPS $h$ and $k$ profiles, a Gaussian profile has been attributed to each component of the system in the fitting procedures. A third Gaussian absorption profile represents the interstellar absorption component (Figure 6). In the Gaussian fitting procedures, since the effect of interstellar absorption can be removed and this removal does not show any significant effect in comparison with the observational errors on flux and wavelength of the IUE images, the uncertainties in the strength and velocity of NEWSIPS $h$ and $k$ lines can be estimated independently based on the observational errors. Therefore, in this analysis we can be confident that there were no effects of interstellar absorption on determination of the secondary's contribution to the activity of the system. The orbital phases that correspond to the mid-time of IUE observations were computed with the ephemeris

$$
\mathrm{HJD}=(2440133.766+6.43791 \times E) \text { days },
$$

for which the zero phase corresponds to conjunction with the primary (K0 IV) component in front (Carlos \& Popper 1971).

\subsection{Short-Wavelength, Low-Dispersion Spectra}

The most prominent feature seen in the spectra is Ly $\alpha$ profile. Since this line is blended with geocoronal Ly $\alpha$ and overexposed throughout the observations, it is not included in the line analysis. Due to the limitations of the 6 - $\AA$ resolution, some of the emission lines are unresolved or partially resolved multiplets. The identified emission lines of SWP spectra (see Figure 1) are OI $(\lambda \lambda 1302,1305)$, CI $(\lambda 1657)$, SiII $(\lambda \lambda 1808,1817)$, CII $(\lambda \lambda 1334,1335)$, HeII $(\lambda 1639)$, Nv $(\lambda 1238)$, Sirv $(\lambda \lambda 1393,1402)$ and Civ $(\lambda \lambda 1548,1550)$. Figures 2 to 4 show the integrated emission Carbon line fluxes of low-dispersion spectra as a function of time (orbital epoch) and orbital phase. These lines originate in chromosphere and transition region. The 
Table 2. The log of IUE observations of UX Ari

\begin{tabular}{|c|c|c|c|c|c|c|c|c|c|}
\hline Image & Disp & $\mathrm{HJD}$ (mid) & $\begin{array}{c}\text { Exposure } \\
\text { time (s) }\end{array}$ & Comment & Image & Disp & $\mathrm{HJD}$ (mid) & $\begin{array}{c}\text { Exposure } \\
\text { time (s) }\end{array}$ & Comment \\
\hline LWR02081 & $\mathrm{H}$ & 2443736.0752 & 720 & * & LWP11752 & $\mathrm{L}$ & 2447068.3635 & 90 & \\
\hline SWP02301 & $\mathrm{L}$ & 2443736.1016 & 2700 & * & LWP11753 & $\mathrm{L}$ & 2447068.4835 & 90 & \\
\hline LWR02082 & $\mathrm{H}$ & 2443736.1354 & 1800 & * & LWP11754 & $\mathrm{L}$ & 2447068.6115 & 90 & \\
\hline LWR02111 & $\mathrm{H}$ & 2443739.8502 & 1800 & $*$ & LWP11755 & $\mathrm{L}$ & 2447068.7315 & 90 & \\
\hline SWP02336 & $\mathrm{L}$ & 2443739.8983 & 5400 & $*$ & LWP11756 & $\mathrm{H}$ & 2447068.7764 & 3000 & \\
\hline LWR02136 & $\mathrm{H}$ & 2443741.9434 & 1800 & $*$ & LWP11757 & $\mathrm{H}$ & 2447068.8277 & 1500 & \\
\hline SWP02351 & $\mathrm{L}$ & 2443741.9853 & 4200 & $*$ & LWP11758 & $\mathrm{H}$ & 2447068.8727 & 1500 & \\
\hline LWR02158 & $\mathrm{H}$ & 2443743.9244 & 1800 & * & LWP11760 & $\mathrm{L}$ & 2447068.9725 & 90 & \\
\hline SWP02375 & $\mathrm{L}$ & 2443743.9643 & 4200 & * & LWP11761 & $\mathrm{L}$ & 2447068.9975 & 90 & \\
\hline LWR03344 & $\mathrm{H}$ & 2443874.5894 & 1800 & $*$ & LWP11762 & $\mathrm{H}$ & 2447069.0424 & 3000 & \\
\hline SWP03766 & $\mathrm{L}$ & 2443874.6273 & 4200 & * & LWP11763 & $\mathrm{L}$ & 2447069.0845 & 90 & \\
\hline SWP03855 & $\mathrm{L}$ & 2443882.7704 & 1800 & $*$ & LWP11764 & $\mathrm{L}$ & 2447069.2335 & 90 & \\
\hline LWR03432 & $\mathrm{H}$ & 2443882.7963 & 1080 & * & LWP11765 & $\mathrm{L}$ & 2447069.3595 & 90 & \\
\hline LWR06261 & $\mathrm{H}$ & 2444207.2292 & 900 & & LWP11766 & $\mathrm{L}$ & 2447069.4825 & 90 & \\
\hline SWP07267 & $\mathrm{L}$ & 2444207.2668 & 4800 & $*$ & LWP11767 & $\mathrm{L}$ & 2447069.5875 & 90 & \\
\hline LWR06329S & $\mathrm{L}$ & 2444215.9747 & 120 & & LWP11768 & $\mathrm{L}$ & 2447069.6135 & 90 & \\
\hline LWR06329L & $\mathrm{L}$ & 2444215.9784 & 240 & & LWP11769 & $\mathrm{L}$ & 2447069.6375 & 90 & \\
\hline SWP07342 & $\mathrm{L}$ & 2444216.0098 & 4800 & * & LWP11770 & $\mathrm{L}$ & 2447069.6635 & 90 & \\
\hline LWR06330 & $\mathrm{H}$ & 2444216.0524 & 1800 & & LWP11771 & $\mathrm{H}$ & 2447069.7233 & 5400 & \\
\hline SWP07423 & $\mathrm{L}$ & 2444225.4259 & 12600 & * & LWP14051 & $\mathrm{H}$ & 2447419.8604 & 3000 & \\
\hline LWR10244 & $\mathrm{H}$ & 2444693.3949 & 1200 & * & LWP14052 & $\mathrm{H}$ & 2447419.9127 & 1500 & \\
\hline SWP13612 & $\mathrm{L}$ & 2444693.4234 & 3000 & $*$ & LWP14053 & $\mathrm{H}$ & 2447419.9472 & 720 & \\
\hline SWP15211 & $\mathrm{H}$ & 2444886.6123 & 27000 & *, noisy, excluded & LWP14084 & $\mathrm{H}$ & 2447422.9887 & 1500 & \\
\hline LWR11729 & $\mathrm{H}$ & 2444886.7817 & 1500 & $*$ & LWP14085 & $\mathrm{H}$ & 2447423.0317 & 1500 & \\
\hline SWP15240 & $\mathrm{H}$ & 2444889.5829 & 24000 & *, noisy, excluded & LWP14086 & $\mathrm{H}$ & 2447423.0926 & 4080 & \\
\hline LWR11756 & $\mathrm{H}$ & 2444889.7319 & 1200 & & LWP14130 & $\mathrm{H}$ & 2447432.8294 & 3000 & \\
\hline SWP26730 & $\mathrm{L}$ & 2446334.6765 & 600 & * & LWP14131 & $\mathrm{H}$ & 2447432.8817 & 1500 & \\
\hline SWP26730 & $\mathrm{L}$ & 2446334.6875 & 600 & $*$ & LWP14132 & $\mathrm{H}$ & 2447432.9334 & 3000 & \\
\hline SWP26730 & $\mathrm{L}$ & 2446334.7095 & 600 & * & LWP14152 & $\mathrm{H}$ & 2447435.9527 & 1500 & \\
\hline LWP06815 & $\mathrm{H}$ & 2446334.7232 & 900 & & LWP14153 & $\mathrm{H}$ & 2447435.9967 & 1500 & \\
\hline SWP26731 & $\mathrm{L}$ & 2446334.7415 & 600 & * & LWP14220 & $\mathrm{H}$ & 2447448.7844 & 3000 & \\
\hline SWP26731 & $\mathrm{L}$ & 2446334.7555 & 600 & $*$ & LWP14221 & $\mathrm{H}$ & 2447448.8367 & 1500 & \\
\hline SWP26731 & $\mathrm{L}$ & 2446334.7675 & 600 & * & LWP14222 & $\mathrm{H}$ & 2447448.8777 & 1500 & \\
\hline LWP06816 & $\mathrm{H}$ & 2446334.7892 & 900 & & LWP14263 & $\mathrm{H}$ & 2447451.9177 & 1500 & \\
\hline SWP26732 & $\mathrm{L}$ & 2446334.8095 & 600 & $*$ & LWP14264 & $\mathrm{H}$ & 2447451.9587 & 1500 & \\
\hline SWP26732 & $\mathrm{L}$ & 2446334.8225 & 600 & * & LWP14265 & $\mathrm{H}$ & 2447452.0141 & 3480 & \\
\hline SWP26732 & $\mathrm{L}$ & 2446334.8345 & 600 & $*$ & LWP18569 & $\mathrm{H}$ & 2448116.5273 & 1080 & \\
\hline LWP06817 & $\mathrm{H}$ & 2446334.8552 & 900 & & SWP39449 & $\mathrm{L}$ & 2448116.5547 & 1500 & \\
\hline SWP26733 & $\mathrm{L}$ & 2446334.8755 & 600 & *, noisy, excluded & SWP39460 & $\mathrm{L}$ & 2448118.0615 & 1806 & \\
\hline SWP26733 & $\mathrm{L}$ & 2446334.8925 & 600 & *, noisy, excluded & LWP18584 & $\mathrm{H}$ & 2448118.0829 & 1200 & \\
\hline SWP26733 & $\mathrm{L}$ & 2446334.9035 & 600 & *, noisy, excluded & SWP39470 & $\mathrm{L}$ & 2448118.9079 & 2400 & \\
\hline LWP06818 & $\mathrm{H}$ & 2446334.9252 & 900 & & LWP18597 & $\mathrm{H}$ & 2448119.8816 & 1320 & \\
\hline SWP26734 & $\mathrm{L}$ & 2446334.9435 & 600 & Noisy, excluded & LWP18607 & $\mathrm{H}$ & 2448121.0516 & 1320 & \\
\hline SWP26734 & $\mathrm{L}$ & 2446334.9575 & 600 & Noisy, excluded & SWP39476 & $\mathrm{L}$ & 2448121.0769 & 2400 & \\
\hline SWP26734 & $\mathrm{L}$ & 2446334.9658 & 144 & Noisy, excluded & SWP42405 & $\mathrm{L}$ & 2448505.9212 & 2100 & \\
\hline LWP06819 & $\mathrm{H}$ & 2446334.9952 & 900 & & LWP21171 & $\mathrm{H}$ & 2448505.9433 & 1080 & \\
\hline SWP26735 & $\mathrm{L}$ & 2446335.0145 & 600 & Noisy, excluded & SWP42416 & $\mathrm{L}$ & 2448507.9249 & 2400 & \\
\hline SWP26735 & $\mathrm{L}$ & 2446335.0265 & 600 & Noisy, excluded & LWP21187 & $\mathrm{H}$ & 2448507.9485 & 600 & \\
\hline LWP09864 & $\mathrm{H}$ & 2446801.4872 & 900 & & LWP21200 & $\mathrm{H}$ & 2448508.9073 & 1080 & \\
\hline SWP30026 & $\mathrm{L}$ & 2446801.5055 & 600 & $*$ & SWP42427 & $\mathrm{L}$ & 2448508.9379 & 2400 & \\
\hline SWP30026 & $\mathrm{L}$ & 2446801.5165 & 600 & $*$ & LWP21208 & $\mathrm{H}$ & 2448509.8913 & 1080 & \\
\hline SWP30026 & $\mathrm{L}$ & 2446801.5285 & 600 & $*$ & SWP42435 & $\mathrm{L}$ & 2448509.9209 & 2400 & \\
\hline LWP09865 & $\mathrm{H}$ & 2446801.5512 & 900 & * & LWP21222 & $\mathrm{H}$ & 2448511.8890 & 1380 & \\
\hline SWP30027 & $\mathrm{L}$ & 2446801.5725 & 600 & $*$ & SWP42448 & $\mathrm{L}$ & 2448511.9179 & 2400 & \\
\hline SWP30027 & $\mathrm{L}$ & 2446801.5825 & 600 & * & LWP21236 & $\mathrm{H}$ & 2448513.8990 & 1380 & \\
\hline SWP30027 & $\mathrm{L}$ & 2446801.5955 & 600 & $*$ & SWP42461 & $\mathrm{L}$ & 2448513.9269 & 2400 & * \\
\hline LWP09866 & $\mathrm{H}$ & 2446801.6172 & 900 & $*$ & LWP28935 & $\mathrm{H}$ & 2449585.1275 & 600 & \\
\hline SWP30028 & $\mathrm{L}$ & 2446801.6375 & 600 & * & SWP51857 & $\mathrm{L}$ & 2449585.1502 & 900 & \\
\hline SWP30028 & $\mathrm{L}$ & 2446801.6485 & 600 & * & SWP51858 & $\mathrm{L}$ & 2449585.1842 & 900 & \\
\hline SWP30028 & $\mathrm{L}$ & 2446801.6615 & 600 & * & LWP28940 & $\mathrm{H}$ & 2449585.9735 & 600 & \\
\hline LWP09867 & $\mathrm{H}$ & 2446801.6852 & 900 & * & SWP51866 & $\mathrm{L}$ & 2449585.9952 & 900 & \\
\hline SWP30029 & $\mathrm{L}$ & 2446801.7045 & 600 & $*$ & SWP51867 & $\mathrm{L}$ & 2449586.0272 & 900 & \\
\hline
\end{tabular}


Table 2. (Continued)

\begin{tabular}{|c|c|c|c|c|c|c|c|c|c|}
\hline Image & Disp & HJD(mid) & $\begin{array}{l}\text { Exposure } \\
\text { time (s) }\end{array}$ & Comment & Image & Disp & HJD(mid) & $\begin{array}{l}\text { Exposure } \\
\text { time (s) }\end{array}$ & Comment \\
\hline SWP30029 & $\mathrm{L}$ & 2446801.7165 & 600 & $*$ & SWP51872 & $\mathrm{L}$ & 2449586.9082 & 900 & \\
\hline SWP30029 & $\mathrm{L}$ & 2446801.7275 & 600 & * & LWP28943 & $\mathrm{H}$ & 2449586.9205 & 600 & \\
\hline LWP09868 & $\mathrm{H}$ & 2446801.7502 & 900 & * & SWP51873 & $\mathrm{L}$ & 2449586.9432 & 900 & \\
\hline SWP30030 & $\mathrm{L}$ & 2446801.7705 & 600 & $*$ & SWP51884 & $\mathrm{L}$ & 2449587.9022 & 900 & \\
\hline SWP30030 & $\mathrm{L}$ & 2446801.7805 & 600 & $*$ & LWP28950 & $\mathrm{H}$ & 2449587.9145 & 600 & \\
\hline LWP11745 & $\mathrm{H}$ & 2447067.8534 & 3000 & & SWP51885 & $\mathrm{L}$ & 2449587.9392 & 900 & \\
\hline LWP11746 & $\mathrm{H}$ & 2447067.9037 & 1500 & & SWP51961 & $\mathrm{L}$ & 2449592.8712 & 900 & \\
\hline LWP11747 & $\mathrm{L}$ & 2447067.9365 & 90 & & LWP29029 & $\mathrm{H}$ & 2449592.8835 & 600 & \\
\hline LWP11748 & $\mathrm{L}$ & 2447067.9595 & 90 & & SWP51962 & $\mathrm{L}$ & 2449592.9092 & 900 & \\
\hline LWP11749 & $\mathrm{H}$ & 2447068.0067 & 1500 & & SWP51975 & $\mathrm{L}$ & 2449593.8772 & 900 & \\
\hline LWP11750 & $\mathrm{H}$ & 2447068.0673 & 4200 & & LWP29040 & $\mathrm{H}$ & 2449593.8895 & 600 & \\
\hline SWP31952 & $\mathrm{H}$ & 2447068.6157 & 89280 & Noisy, excluded & SWP51976 & $\mathrm{L}$ & 2449593.9112 & 900 & \\
\hline LWP11751 & $\mathrm{L}$ & 2447068.2375 & 90 & & LWP29052 & $\mathrm{H}$ & 2449594.9765 & 600 & \\
\hline SWP51986 & $\mathrm{L}$ & 2449594.9992 & 900 & & SWP52056 & $\mathrm{L}$ & 2449602.9102 & 900 & \\
\hline SWP51996 & $\mathrm{L}$ & 2449597.0522 & 900 & & LWP29118 & $\mathrm{H}$ & 2449602.9275 & 600 & \\
\hline LWP29070 & $\mathrm{H}$ & 2449597.0695 & 600 & & SWP52057 & $\mathrm{L}$ & 2449602.9492 & 900 & \\
\hline SWP51997 & $\mathrm{L}$ & 2449597.0922 & 900 & & LWP29127 & $\mathrm{H}$ & 2449604.0545 & 600 & \\
\hline LWP29071 & $\mathrm{H}$ & 2449597.1125 & 600 & & SWP52063 & $\mathrm{L}$ & 2449604.0772 & 900 & \\
\hline LWP29077 & $\mathrm{H}$ & 2449598.0515 & 600 & & LWP29128 & $\mathrm{H}$ & 2449604.1025 & 600 & \\
\hline SWP52007 & $\mathrm{L}$ & 2449598.0742 & 900 & & LWP29137 & $\mathrm{H}$ & 2449605.0615 & 600 & \\
\hline LWP29078 & $\mathrm{H}$ & 2449598.0945 & 600 & & SWP52070 & $\mathrm{L}$ & 2449605.0872 & 900 & \\
\hline SWP52008 & $\mathrm{L}$ & 2449598.1135 & 600 & & LWP29138 & $\mathrm{H}$ & 2449605.1145 & 600 & \\
\hline SWP52016 & $\mathrm{L}$ & 2449599.0652 & 900 & & SWP52078 & $\mathrm{L}$ & 2449606.0732 & 900 & \\
\hline LWP29085 & $\mathrm{H}$ & 2449599.0765 & 600 & & LWP29142 & $\mathrm{H}$ & 2449606.0925 & 600 & \\
\hline SWP52017 & $\mathrm{L}$ & 2449599.0992 & 900 & & LWP29149 & $\mathrm{H}$ & 2449606.8835 & 600 & \\
\hline SWP52023 & $\mathrm{L}$ & 2449600.0622 & 900 & & SWP52086 & $\mathrm{L}$ & 2449606.9052 & 900 & Noisy, exclude \\
\hline LWP29091 & $\mathrm{H}$ & 2449600.0805 & 600 & & SWP52087 & $\mathrm{L}$ & 2449606.9342 & 900 & Noisy, exclude \\
\hline SWP52024 & $\mathrm{L}$ & 2449600.1012 & 900 & & LWP31888 & $\mathrm{L}$ & 2450100.3785 & 90 & \\
\hline SWP52034 & $\mathrm{L}$ & 2449601.0572 & 900 & & SWP56587 & $\mathrm{L}$ & 2450100.4143 & 5400 & $*$ \\
\hline LWP29099 & $\mathrm{H}$ & 2449601.0735 & 600 & & LWP31894 & $\mathrm{L}$ & 2450103.3545 & 90 & \\
\hline SWP52035 & $\mathrm{L}$ & 2449601.0942 & 900 & & SWP56624 & $\mathrm{L}$ & 2450103.3913 & 5400 & \\
\hline LWP29100 & $\mathrm{H}$ & 2449601.1135 & 600 & & LWP31895 & $\mathrm{L}$ & 2450103.3885 & 90 & \\
\hline SWP52046 & $\mathrm{L}$ & 2449602.0722 & 900 & & LWP31896 & $\mathrm{L}$ & 2450103.4245 & 90 & \\
\hline LWP29109 & $\mathrm{H}$ & 2449602.0895 & 600 & & SWP56630 & $\mathrm{L}$ & 2450105.3971 & 4680 & \\
\hline SWP52047 & $\mathrm{L}$ & 2449602.1092 & 900 & & LWP31903 & $\mathrm{L}$ & 2450105.3995 & 90 & \\
\hline
\end{tabular}

${ }^{\dagger}$ Images studied by other authors (see http://archive.stsci.edu/iue/search.php) in the past.

same trend was seen for the other line fluxes (the lines mentioned above).

There is a flare event near the orbital phase $\sim 0.07$ (SWP03766). Apart from this flare event there are two rises in flux at phases $\sim 0.20$ and $\sim 0.70$ (Figures $2-4$ ). It can be clearly seen that there are variations in the chromospheric and transition-region line fluxes with time and orbital phase. Since some of these low dispersion spectra (five images) are very noisy and have indeterminate lines, they were excluded from the analysis. These images are SWP26733, SWP26734, SWP26735, SWP52086 and SWP52087. The scattering of the emission line fluxes of the images, taken at the same epoch (see Figures 2-4) arose from the variation of flux with orbital phase.

\subsection{Short-Wavelength, High-Dispersion Spectra}

There exist only three images of UX Ari system taken by the SWP camera in high resolution: SWP15211, SWP15240 and SWP31952. Nv ( $\lambda 1239)$, OI $(\lambda \lambda 1302,1305)$, CiI $(\lambda \lambda 1334,1335)$, SiIV $(\lambda \lambda 1394,1403)$,
CIV $(\lambda \lambda 1548,1550)$, HeII $(\lambda 1640)$, CI $(\lambda 1657)$, SiII $(\lambda \lambda 1808,1817)$, SiIII $(\lambda 1892)$ and CIII $(\lambda 1909)$ lines were looked for in these three images to evaluate the fluxes of these lines together with those of the low dispersion spectra. Unfortunately, all three images have a lot of reseau (in the ITF), permanent ITF artifact, saturated pixels, warning tracks, RAW-SCREEN cosmic rays/bright spots, positively extrapolated ITF, and very noisy data in the range of these lines. Hence, these lines were hardly seen in the images SWP15211, SWP15240 and SWP31952, which were taken at orbital epochs $738.259,738.721$ and 1077.190 , respectively. Therefore, these three images were excluded from the analysis.

\subsection{Long-Wavelength, Low-Dispersion Spectra}

The long-wavelength low dispersion spectra (24 images) were examined for ultraviolet excess, by comparing the ultraviolet continuum level of UX Ari (G5 V+K0 IV) with the levels of $\kappa$ Cet (G5 V) and $\eta$ Cep (K0 IV) in the same spectral range (between $2100 \AA$ and $3200 \AA$ ). After 

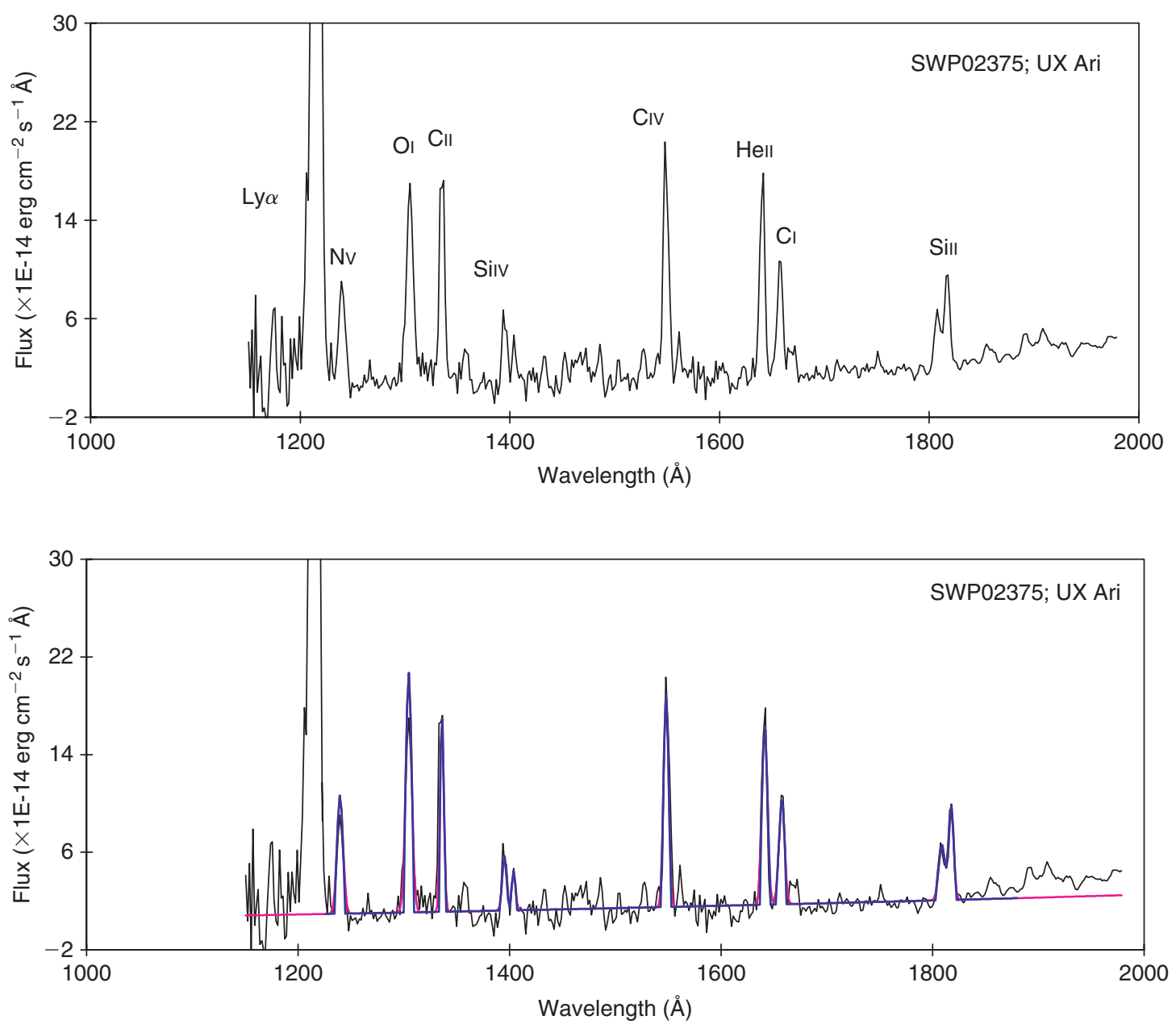

Figure 1 The Gaussian fits to chromospheric and transition-region lines of UX Ari appeared in the SWP02375 low-dispersion image.

polynomial fitting for the continuum levels (see Figure 5) and computing the integrated flux measured on Earth $(f)$ between $2100 \AA$ and $3200 \AA$, the continuum level analysis can be carried out by converting the flux measured on Earth to the surface integrated flux $(F)$ of a star by:

$$
F=\left(\frac{d}{R}\right)^{2} f
$$

where $d$ is the distance from Earth and $R$ is the radius of the star (Gray 1992). If $R$ is in units of solar radii and $d$ is in $\mathrm{pc}$, this relation can be written as

$$
F=1.96249 \times 10^{15}\left(\frac{d}{R}\right)^{2} f .
$$

Equation (2) can be written as

$$
\frac{F}{f}=\left(\frac{d}{R}\right)^{2}=\left(\frac{2}{\Theta}\right)^{2},
$$

where $\Theta=2 R / d$ is the angular diameter of the star, in radians. For double-lined uneclipsing binary stars, like UX Ari, $\Theta$ could be taken as $\Theta=2\left(R_{\mathrm{a}}+R_{\mathrm{b}}\right)^{2} / d$. Here $R_{a}$ and $R_{b}$ are the radius of the compoment stars of a binary system. Since $\left(R_{\mathrm{a}}+R_{\mathrm{b}}\right) \ll d$,
$\left(R_{\mathrm{a}}+R_{\mathrm{b}}\right)^{2} \approx\left(R_{\mathrm{a}}\right)^{2}+\left(R_{\mathrm{b}}\right)^{2}$. Then, Equation (2) can be written for binary system as

$$
F=\left(\frac{d^{2}}{R_{\mathrm{a}}^{2}+R_{\mathrm{b}}^{2}}\right) f .
$$

The distance of UX Ari is given as $50 \mathrm{pc}$ by Strassmeier et al. (1988) and Strassmeier et al. (1993). The recent and most reliable measurement of the distance $(50.23 \mathrm{pc})$ is given in the Hipparcos Catalogue (Perryman et al. 1997). Since the standard error of the parallax of UX Ari is 1.25 mas, given by Perryman et al. (1997), the distance of $50 \mathrm{pc}$ can be adopted as a good estimate.

There are three remarkable estimations on the radii of the components of UX Ari. Therefore, three conditions could be taken into consideration for UX Ari system:

1. With a distance of $50 \mathrm{pc}$ and component radii $R_{\mathrm{G} 5}=0.93 R_{\odot}$ and $R_{\mathrm{K} 0}=3 R_{\odot}$ (Strassmeier et al. 1988), Equation (3) gives:

$$
F=4.906225 \times 10^{17} f
$$

where $R^{2}=R_{\mathrm{G} 5}^{2}+R_{\mathrm{K} 0}^{2}$ for the UX Ari system.

2. With the same distance as given in (1), but radii $R_{\mathrm{G} 5}=$ $0.93 R_{\odot}$ and $R_{\mathrm{K} 0}=4.7 R_{\odot}$ (Strassmeier et al. 1993), 

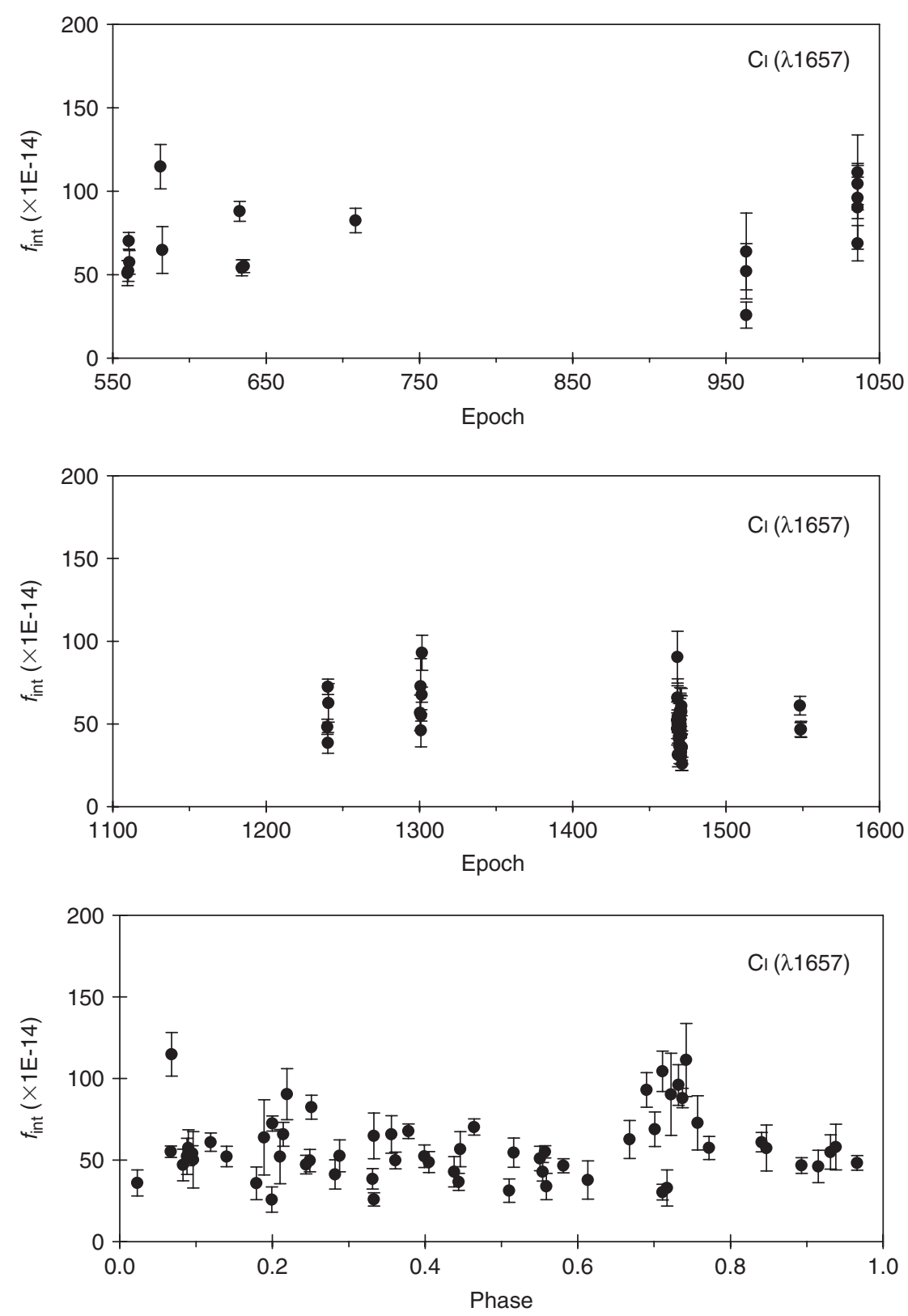

Figure 2 Low dispersion CI emission line (originates in the middle chromosphere) fluxes as a function of time and orbital phase. The line fluxes are in units of $\mathrm{erg} \mathrm{cm}^{-2} \mathrm{~s}^{-1}$.

this relation becomes:

$$
F=2.137332334 \times 10^{17} f
$$

3. With a distance of $50 \mathrm{pc}$ and radii $R_{\mathrm{G} 5}=0.80 R_{\odot}$, $R_{\mathrm{K} 0}=6.2 R_{\odot}$ (Huenemoerder et al. 1989), this relation is

$$
F=1.255431167 \times 10^{17} f .
$$

By using Equations 4, 5 and 6, the integrated surface fluxes of the UV continuum were computed for each aspect given above, between 2100 and $3200 \AA$.

For the comparison stars, $\eta$ Cep and $\kappa$ Cet, this relation can be obtained as follows. At a distance of $14.34 \mathrm{pc}$ (Perryman et al. 1997) and the radius of $R=4 R_{\odot}$ (Blackwell \& Lynas-Gray 1994) for $\eta$ Cep (K0 IV)
Equation 3 gives:

$$
F=2.522236304 \times 10^{16} f
$$

and, with the distance of $9.16 \mathrm{pc}$ (Perryman et al. 1997) and the radius of $R=0.9313 R_{\odot}$ (Blackwell \& Lynas-Gray 1994) for the $\kappa$ Cet (G5 V) Equation 3 gives:

$$
F=1.898537562 \times 10^{17} f .
$$

The integrated continuum fluxes measured on Earth and corresponding surface fluxes between 2100 and $3200 \AA$ spectral range obtained by means of the relations given above are listed in Table 3 for UX Ari system, and in Table 4 for $\eta$ Cep and $\kappa$ Cet together with IUE images. It can be seen that the fluxes obtained from LWR04857S 

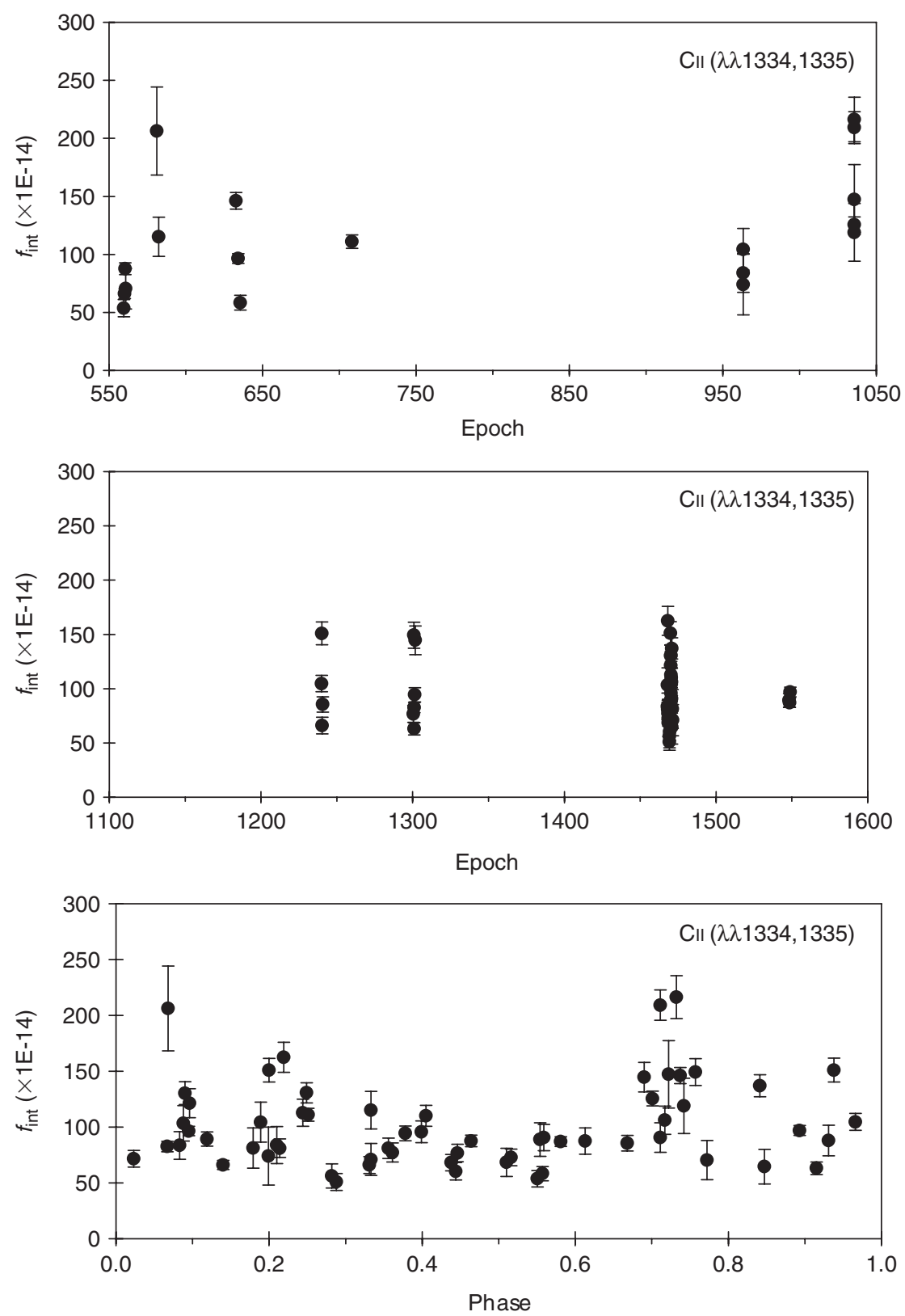

Figure 3 Low dispersion CII emission line (originates in the upper chromosphere) fluxes as a function of time and orbital phase. The line fluxes are in units of $\mathrm{erg} \mathrm{cm}^{-2} \mathrm{~s}^{-1}$.

are much lower than those obtained from the other two images. The reason is that the LWR04857S image was taken by using small aperture of the spectrograph while others were taken by using large aperture.

The effective temperatures and the radii of comparison stars, $\eta$ Cep and $\kappa$ Cet, are comparible with those of the component stars of UX Ari. Namely,

- $\eta \operatorname{Cep}\left(\mathrm{K} 0\right.$ IV), $T_{\mathrm{e}}=4967 \mathrm{~K}$ (Soubrian et al. 2008);

- UX Ari(K0 IV), $T_{\mathrm{e}}=4750 \mathrm{~K}$ (Vogt \& Hatzes 1991);

- $\eta \mathrm{Cep}\left(\mathrm{K} 0\right.$ IV), $R=4 R_{\odot}$ (Blackwell \& Lynas-Gray 1994);

- UX Ari(K0 IV), $R=4.7 R_{\odot}$ (Strassmeier et al. 1993);

- $\kappa \operatorname{Cet}(\mathrm{G} 5 \mathrm{~V}), T_{\mathrm{e}}=5708 \mathrm{~K}$ (Soubrian et al. 2008);

- UX Ari(G5 V), $T_{\mathrm{e}}=5700 \mathrm{~K}$ (Vogt \& Hatzes 1991);
- $\kappa \operatorname{Cet}(\mathrm{G} 5 \mathrm{~V}), R=0.93 R_{\odot}($ Blackwell \& Lynas-Gray 1994);

- UX Ari(G5 V), $R=0.93 R_{\odot}$ (Strassmeier et al. 1993).

Therefore, recalling that the sum of the fluxes of two components can be used in computing the magnitude of a binary system, like UX Ari, by

$$
m_{1}-m_{\mathrm{s}}=-2.5 \log \left(\frac{f_{1}}{f_{1}+f_{2}}\right),
$$

where the subscripts ' 1 ' and ' 2 ' refer to the component stars, and 's' refers to the system, the theoretical continuum flux of the UX Ari system can be estimated by adjusting for its distance of $50 \mathrm{pc}$ and using the observed continuum 

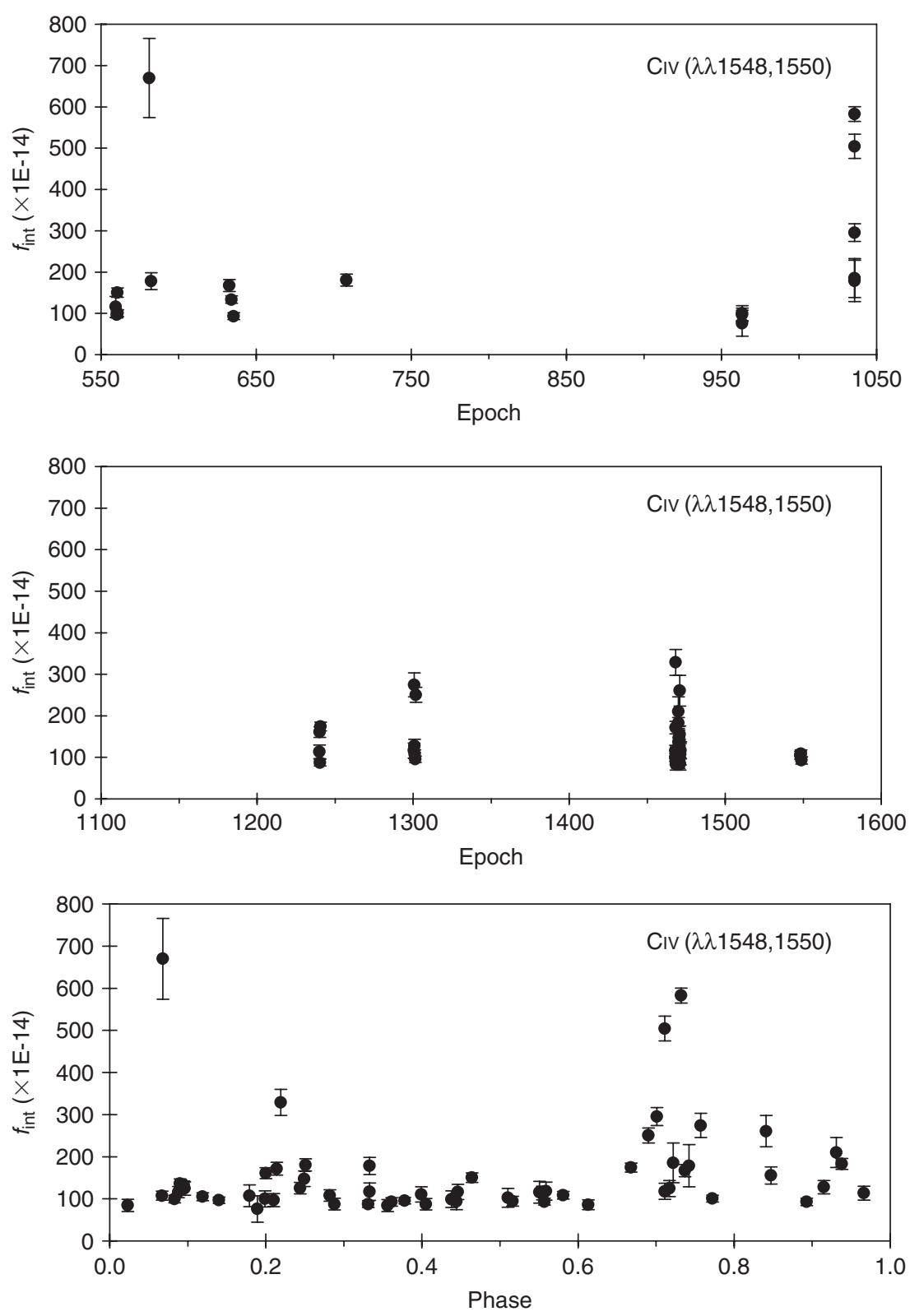

Figure 4 Low dispersion CIv emission line (originates in the transition region) fluxes as a function of time and orbital phase. The line fluxes are in units of $\mathrm{erg} \mathrm{cm}^{-2} \mathrm{~s}^{-1}$.

fluxes of $\eta \operatorname{Cep}(\mathrm{K} 0 \mathrm{IV})$ and $\kappa \operatorname{Cet}(\mathrm{G} 5 \mathrm{~V})$ :

$$
f_{\text {theo, Ari }}=f_{\eta \text { Cep }}+f_{\kappa \text { Cet }} \text {. }
$$

This theoretical flux can be used for the examination of the ultraviolet excess of UX Ari by comparison with the observed continuum fluxes. Here, the theoretical surface fluxes of the comparison stars (see Column 5 of Table 4), $\kappa$ Cet and $\eta$ Cep, were used for evaluating the observed fluxes with adjustment made for the distance of UX Ari. The comparison of observed fluxes (see Column 4 of Table 3) with those of $50 \mathrm{pc}$ observed fluxes (adjustment to the distance of UX Ari, see the last column of Table 4) of $\kappa$ Cet and $\eta$ Cep show that there is some UV excess in UX Ari, which varies from $1 \%$ up to $24 \%$, with the exception of two images (namely LWP31894 and LWP31895). In this evaluation LWR06329S image was excluded because it was taken with the small aperture of the spectrograph. The adjustment $50 \mathrm{pc}$-observed fluxes of comparison stars were computed using the corresponding surface fluxes given in the Column 5 of Table 4.

\subsection{Another Test of the UV Excess in UX Ari}

Although surface fluxes are the most important data in this spectral analysis, it is also useful to look at the result of the testing of the UV excess by taking only the observed fluxes at Earth into consideration, as in the spectrophotometric analysis of Rhombs \& Fix (1977).

By using Equation 12 written as

$$
f_{\text {theo, } \mathrm{UX} \text { Ari }}=C_{1} f_{\eta \text { Cep }}+C_{2} f_{\kappa \text { Cet }},
$$




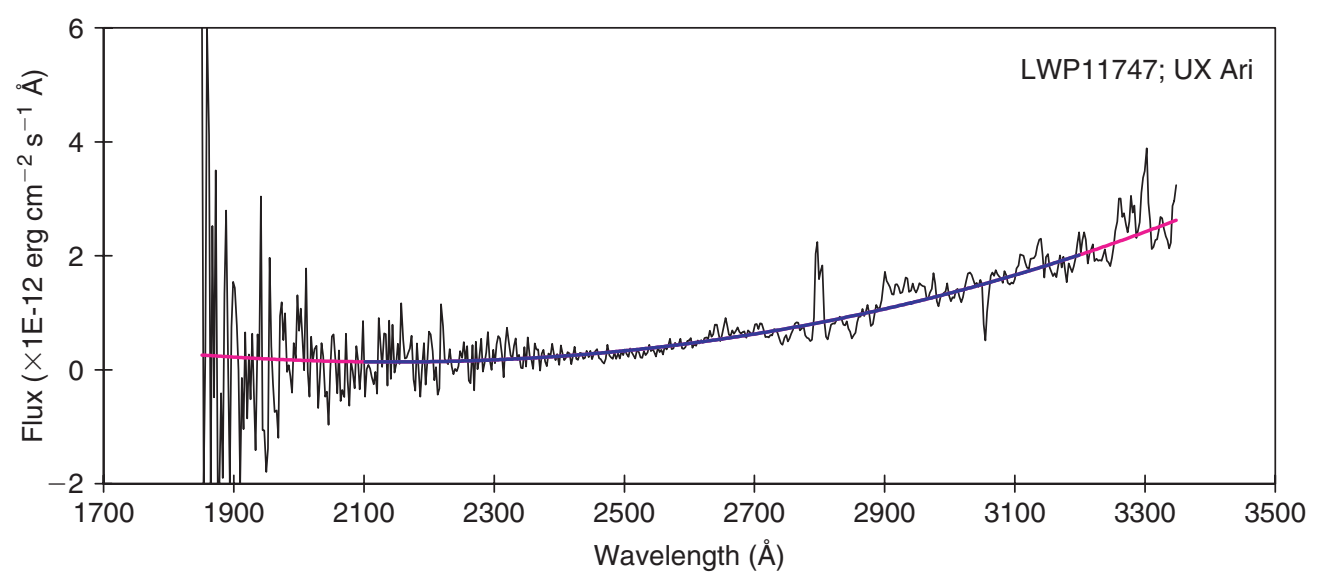

Figure 5 A sample of the third-order polynomial fitting to the UV continuum of the long-wavelength, low-dispersion IUE spectrum, LWP11747.

Table 3. Integrated continuum fluxes

\begin{tabular}{|c|c|c|c|c|c|c|c|}
\hline Image & HJD(mid) & Epoch & $\begin{array}{c}f \\
\left(\times 10^{-10}\right)\end{array}$ & $\begin{array}{l}\text { UV excess } \\
\text { in } f(\%)\end{array}$ & $\begin{array}{c}F_{\text {Str88 }} \\
\left(10^{7} \mathrm{erg} \mathrm{cm}^{-2} \mathrm{~s}^{-1}\right)\end{array}$ & $\begin{array}{c}F_{\text {Huen89 }} \\
\left(10^{7} \mathrm{erg} \mathrm{cm}^{-2} \mathrm{~s}^{-1}\right)\end{array}$ & $\begin{array}{c}F_{\mathrm{Str} 93} \\
\left(10^{7} \mathrm{erg} \mathrm{cm}^{-2} \mathrm{~s}^{-1}\right)\end{array}$ \\
\hline LWR06329S & 2444215.975 & 634.089 & $1.44(0.06)$ & - & $7.07(0.28)$ & $1.81(0.07)$ & $3.08(0.12)$ \\
\hline LWR06329L & 2444215.978 & 634.090 & $2.57(0.05)$ & 4.3 & $12.60(0.24)$ & $3.22(0.06)$ & $5.49(0.11)$ \\
\hline LWP11747 & 2447067.937 & 1077.084 & $2.97(0.05)$ & 20.6 & $14.57(0.26)$ & $3.73(0.07)$ & $6.35(0.11)$ \\
\hline LWP11748 & 2447067.960 & 1077.088 & $3.05(0.05)$ & 24.0 & $14.98(0.26)$ & $3.83(0.07)$ & $6.53(0.12)$ \\
\hline LWP11751 & 2447068.238 & 1077.131 & $2.91(0.07)$ & 18.4 & $14.30(0.32)$ & $3.66(0.08)$ & $6.23(0.14)$ \\
\hline LWP11752 & 2447068.364 & 1077.150 & $2.89(0.06)$ & 17.4 & $14.18(0.27)$ & $3.63(0.07)$ & $6.18(0.12)$ \\
\hline LWP11753 & 2447068.484 & 1077.169 & $2.83(0.05)$ & 15.1 & $13.90(0.27)$ & $3.56(0.07)$ & $6.06(0.12)$ \\
\hline LWP11754 & 2447068.612 & 1077.189 & $2.84(0.05)$ & 15.3 & $13.92(0.27)$ & $3.56(0.07)$ & $6.07(0.12)$ \\
\hline LWP11755 & 2447068.732 & 1077.208 & $2.79(0.06)$ & 13.5 & $13.71(0.27)$ & $3.51(0.07)$ & $5.97(0.12)$ \\
\hline LWP11760 & 2447068.973 & 1077.245 & $2.81(0.06)$ & 14.2 & $13.79(0.27)$ & $3.53(0.07)$ & $6.01(0.12)$ \\
\hline LWP11761 & 2447068.998 & 1077.249 & $2.86(0.05)$ & 16.0 & $14.01(0.24)$ & $3.59(0.06)$ & $6.10(0.11)$ \\
\hline LWP11763 & 2447069.085 & 1077.262 & $2.86(0.06)$ & 16.0 & $14.02(0.27)$ & $3.59(0.07)$ & $6.11(0.12)$ \\
\hline LWP11764 & 2447069.234 & 1077.286 & $2.88(0.05)$ & 16.9 & $14.12(0.27)$ & $3.61(0.07)$ & $6.15(0.12)$ \\
\hline LWP11765 & 2447069.360 & 1077.305 & $2.92(0.06)$ & 18.6 & $14.33(0.27)$ & $3.67(0.07)$ & $6.24(0.12)$ \\
\hline LWP11766 & 2447069.483 & 1077.324 & $2.84(0.05)$ & 15.3 & $13.92(0.27)$ & $3.56(0.07)$ & $6.07(0.12)$ \\
\hline LWP11767 & 2447069.588 & 1077.341 & $2.93(0.06)$ & 19.1 & $14.39(0.28)$ & $3.68(0.07)$ & $6.27(0.12)$ \\
\hline LWP11768 & 2447069.614 & 1077.345 & $2.93(0.06)$ & 19.1 & $14.38(0.28)$ & $3.68(0.07)$ & $6.27(0.12)$ \\
\hline LWP11769 & 2447069.638 & 1077.348 & $2.87(0.06)$ & 16.5 & $14.08(0.28)$ & $3.60(0.07)$ & $6.13(0.12)$ \\
\hline LWP11770 & 2447069.664 & 1077.352 & $2.95(0.06)$ & 19.7 & $14.46(0.29)$ & $3.70(0.07)$ & $6.30(0.13)$ \\
\hline LWP31888 & 2450100.379 & 1548.113 & $2.51(0.05)$ & 1.8 & $12.29(0.23)$ & $3.15(0.06)$ & $5.35(0.10)$ \\
\hline LWP31894 & 2450103.355 & 1548.575 & $2.32(0.05)$ & - & $11.39(0.23)$ & $2.92(0.06)$ & $4.96(0.10)$ \\
\hline LWP31895 & 2450103.389 & 1548.581 & $2.39(0.05)$ & - & $11.72(0.23)$ & $3.00(0.06)$ & $5.11(0.10)$ \\
\hline LWP31896 & 2450103.425 & 1548.586 & $2.48(0.05)$ & 1.0 & $12.17(0.24)$ & $3.11(0.06)$ & $5.30(0.10)$ \\
\hline LWP31903 & 2450105.400 & 1548.893 & $2.55(0.05)$ & 3.4 & $12.49(0.23)$ & $3.20(0.06)$ & $5.44(0.10)$ \\
\hline
\end{tabular}

Table 4. Integrated continuum fluxes of $\eta$ Cep and $\kappa$ Cet

\begin{tabular}{lccccc}
\hline Image & HJD(mid) & Star & $\begin{array}{c}f \\
\left(10^{-10} \mathrm{erg} \mathrm{cm}^{-2} \mathrm{~s}^{-1}\right)\end{array}$ & $\begin{array}{c}F \\
\left(10^{7} \mathrm{erg} \mathrm{cm}^{-2} \mathrm{~s}^{-1}\right)\end{array}$ & $\begin{array}{c}f_{50} \\
\left(10^{-10} \mathrm{erg} \mathrm{cm}^{-2} \mathrm{~s}^{-1}\right)\end{array}$ \\
\hline LWR12739 & 2445037.093 & $\eta$ Cep & $23.32(0.18)$ & $5.88(0.04)$ & $1.92(0.01)$ \\
LWR04857S & 2444048.950 & $\kappa$ Cet & $10.56(0.14)$ & $20.06(0.26)$ & $0.35(0.05)$ \\
LWR04857L & 2444048.955 & $\kappa$ Cet & $17.13(0.16)$ & $32.52(0.29)$ & $0.57(0.05)$ \\
LWR04858 & 2444048.982 & $\kappa$ Cet & $15.29(0.82)$ & $29.03(0.16)$ & $0.51(0.03)$ \\
\hline
\end{tabular}

where

$$
\begin{aligned}
C_{1} & =\left(\frac{R_{\mathrm{K} 0, \mathrm{UX} \text { Ari }}}{R_{\eta} \text { Cep }}\right)^{2}\left(\frac{d_{\eta \text { Cep }}}{d_{\mathrm{UX} \text { Ari }}}\right)^{2} \\
& =0.11356226,
\end{aligned}
$$

with $R_{\mathrm{K} 0, \mathrm{UX} \text { Ari }}=4.7 R_{\odot}$ and

$$
\begin{aligned}
C_{2} & =\left(\frac{R_{\mathrm{G} 5, \mathrm{UX} \text { Ari }}}{R_{\kappa} \mathrm{Cet}}\right)^{2}\left(\frac{d_{\kappa \mathrm{Cet}}}{d_{\mathrm{UX} \mathrm{Ari}}}\right)^{2} \\
& =0.033468606,
\end{aligned}
$$



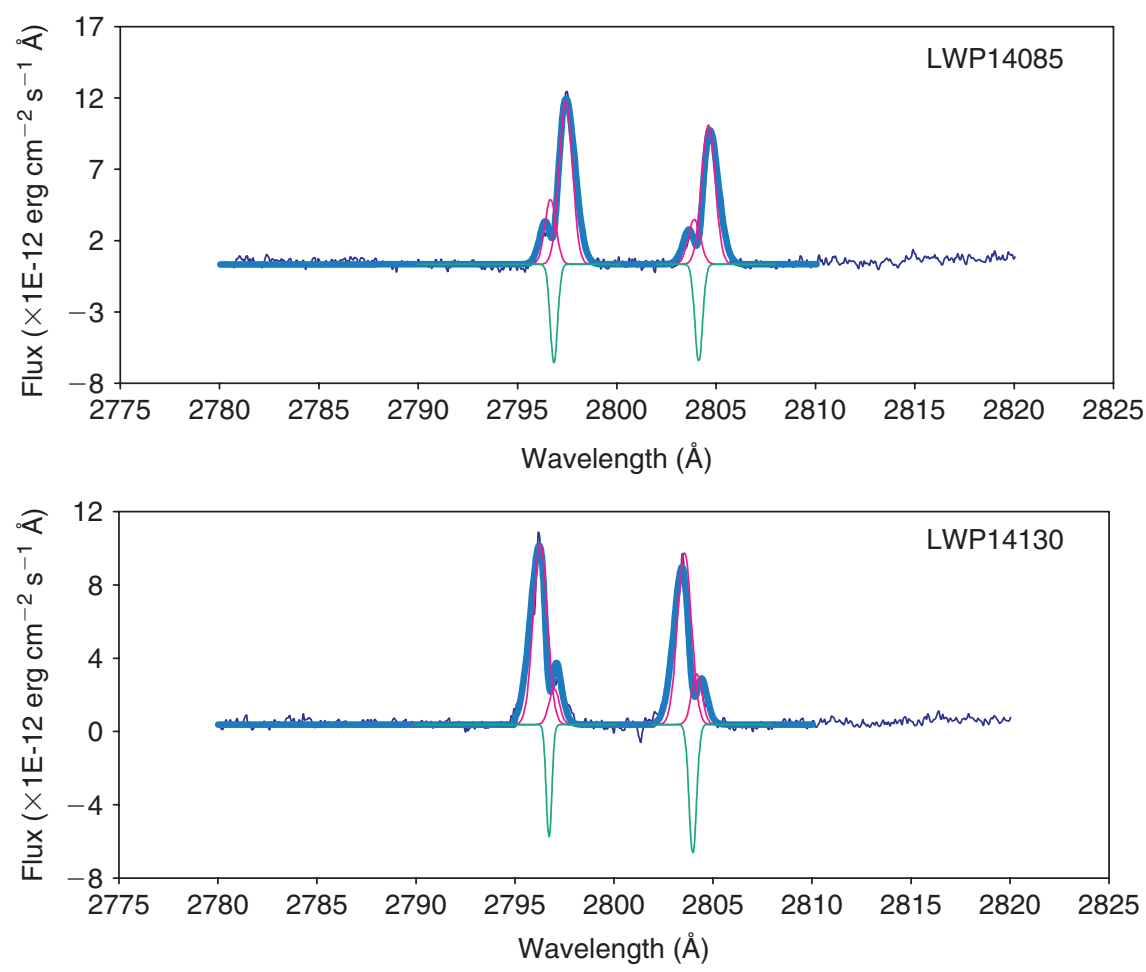

Figure 6 The Gaussian fits to MgII $h$ and $k$ lines of the double-lined spectroscopic binary UX Ari appeared in LWP14085 and LWP14130 high resolution images. The explanation for all components of Gaussian profiles are given in Section 2.

with $R_{\mathrm{K} 0, \mathrm{UX} \text { Ari }}=0.93 R_{\odot}$. Using the values of integrated fluxes measured at Earth of $1.92 \times 10^{-10}$ for $\eta$ Cep and of $0.54 \times 10^{-10}$ (mean value) for $\kappa$ Cet (see Table 4 ), we have

$$
f_{\text {theo, Ari }}=f_{\eta \text { Cep }}+f_{\kappa \text { Cet }}=2.46 \times 10^{-10} .
$$

By examination of the ratio $f_{\mathrm{UX} \text { Ari }} / f_{\text {theo, Ari }}=1.01$ to 1.24. Depending on wavelength, the calculation using the observed UV fluxes at Earth in Equation 12 (second approach) shows also that there is some ultraviolet excess in the UX Ari system.

\subsection{Long-Wavelength, High-Dispersion Spectra}

The most prominent features seen in these spectra are the well-known chromospheric MgII $h$ and $k$ emission lines. In all LWP and LWR high resolution images, these MgII $h$ and $k$ line profiles of both K0 IV and G5 V stars, appeared to be compatible with the corresponding orbital phases (Figures 6 and 7). Therefore, the NEwsiPs $h$ and $k$ flux variation can also be evaluated depending on orbital phase. Based on the fitting procedure mentioned at the beginning of Section 2, the integrated line fluxes, the equivalent widths and the radial velocities for all components of the MgII profiles were computed. The integrated line fluxes of MgII $k$ and MgII $h$ and $k$, from G5 V and K0 IV component, are plotted in Figures 8 and 9 as a function of time (in the sense of epoch) and orbital phase, respectively. Similar trends were seen for the total MgII line fluxes of both components (G5 V+K0 IV) of UX Ari. The scattering of MgII line fluxes that appeared in Figure 8 is similar to that in Figures 2-4. This scattering was also attributed to the flux variation (which showed the maxima near 0.20 and 0.70 orbital phases) with the orbital phase. The MgII $h$ and $k$ radial velocity curves of UX Ari system are shown in Figure 10 together with the results of Duemmler \& Aarum (2001) and of Carlos \& Popper (1971) obtained from the visible spectral range. It is seen that the velocities of K0 IV component are in a better agreement with the optical data than the velocities of G5 V component. Recalling that the effect of interstellar absorption has been removed by the Gaussian profile fitting procedures (see Section 2), this greater scattering in the $\mathrm{G} 5 \mathrm{~V}$ velocities is likely due to the physical interaction between the K0 IV and G5 V component of UX Ari, just as the mass exchange via coronal/ magnetic loops. The velocity $\gamma$ of the centre of mass of the system was found to be $38.22 \pm 2.36 \mathrm{~km} \mathrm{~s}^{-1}$ from sinusoidal fitting to the MgII $h$ radial-velocity curve and $31.01 \pm 2.44 \mathrm{~km} \mathrm{~s}^{-1}$ from fitting the same to the $\operatorname{MgII}_{k}$ radial-velocity curve. Therefore, the mean value of $\gamma$ is found to be $34.62 \pm 4.86 \mathrm{~km} \mathrm{~s}^{-1}$ from the MgII $h$ and $k$ radial velocity curves of UX Ari. This $\gamma$ is somewhat greater (about $8 \mathrm{~km} \mathrm{~s}^{-1}$ ) than that of Duemmler \& Aarum (2001) and Carlos \& Popper (1971, $26.5 \mathrm{~km} \mathrm{~s}^{-1}$ ).

\section{Discussion and Conclusions}

The conclusions of this study together with related discussion are as follows:

All integrated emission line fluxes of short wavelength low dispersion spectra showed a clear variation with time and orbital phase, but the variation with time was not as clear as that with the orbital phase (Figures 2 to 4 ). For 

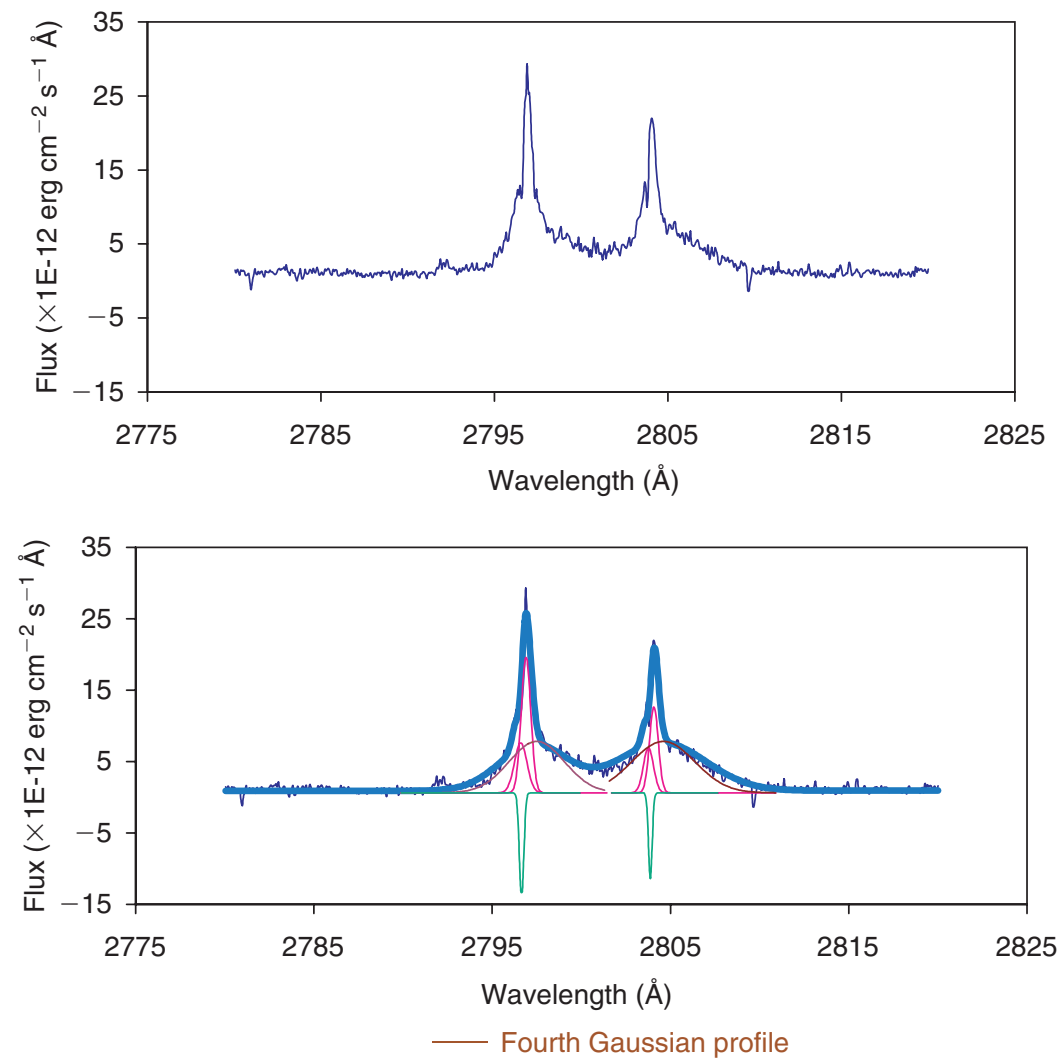

Figure 7 The IUE flare spectrum of UX Ari observed on 1979 Jan 1 at 0.062 orbital phase. In the Gaussian profiles fisting procedure the fourth component was added for the flare effect on the MgII $h$ and $k$ profiles.
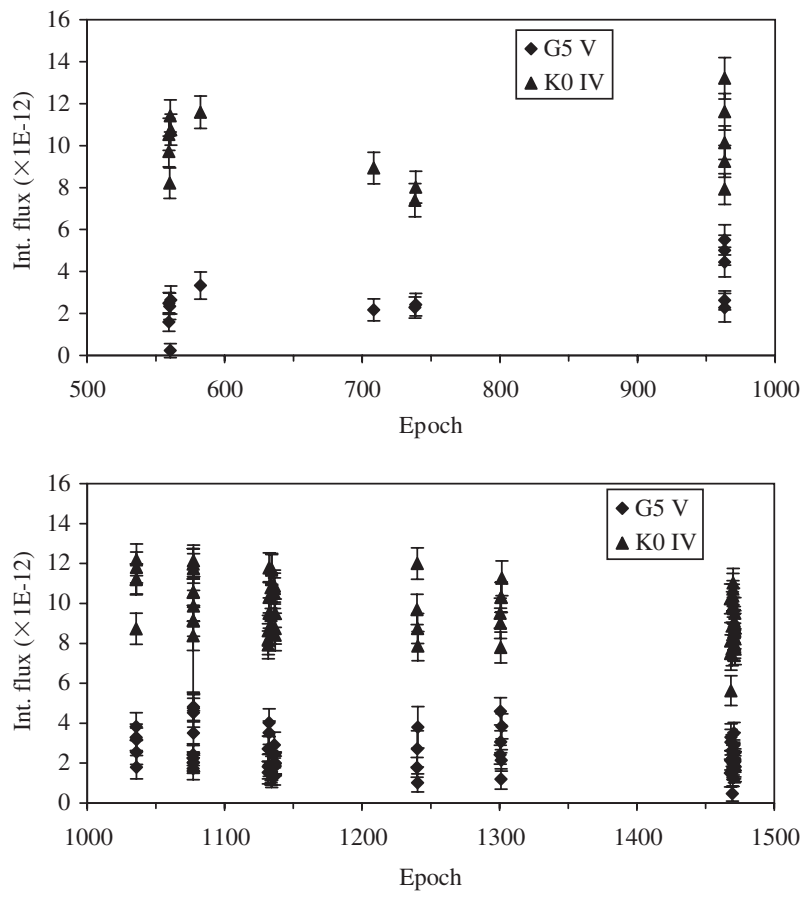

Figure 8 The integrated MgII $k$ line fluxes of the components of UX Ari system as a function of time. The fluxes are in units of $\operatorname{erg} \mathrm{cm}^{-2} \mathrm{~s}^{-1}$.

example, the spectra taken in 1978 (near the epoch of 560) showed a bit larger scattering in the range of fluxes. But when plotting these data versus orbital phase, the flux distribution showed a more clear flux variation. This feature
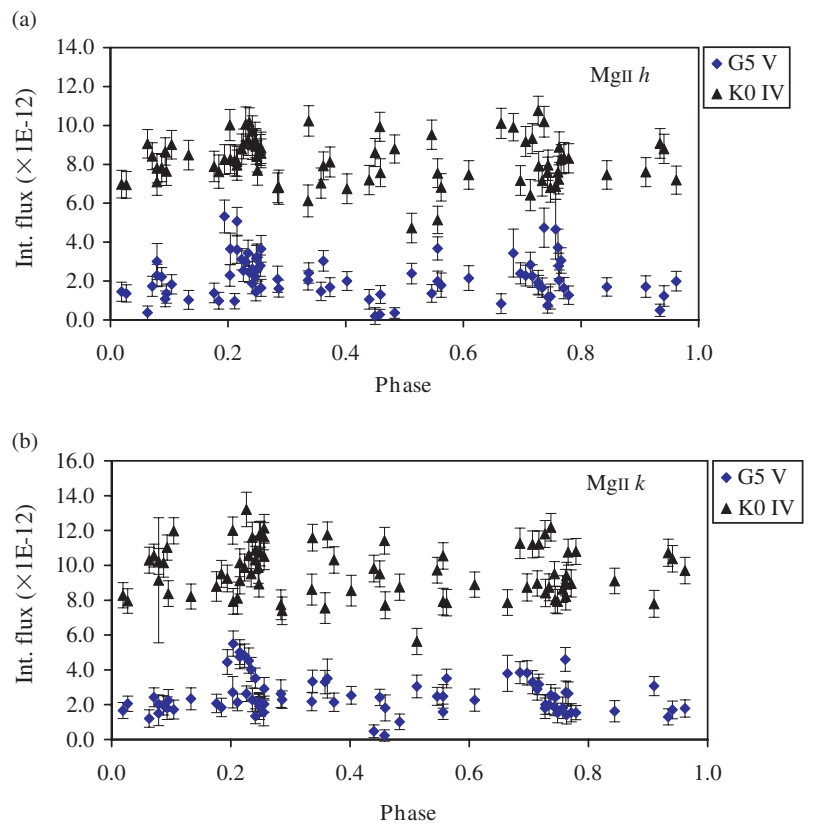

Figure 9 (a) The MgII $h$ line fluxes, and (b) the MgII $k$ line fluxes of the components of UX Ari system as a function of orbital phase. The fluxes are in units of $\mathrm{erg} \mathrm{cm}^{-2} \mathrm{~s}^{-1}$.

seems to be similar in the other epochs (epochs of 963 , 1035,1301 and 1468). Apart from the flare event shown on 1979 Jan 1 (SWP03766), there were some flux enhancements (especially in the lines originating in the middle 
(a)

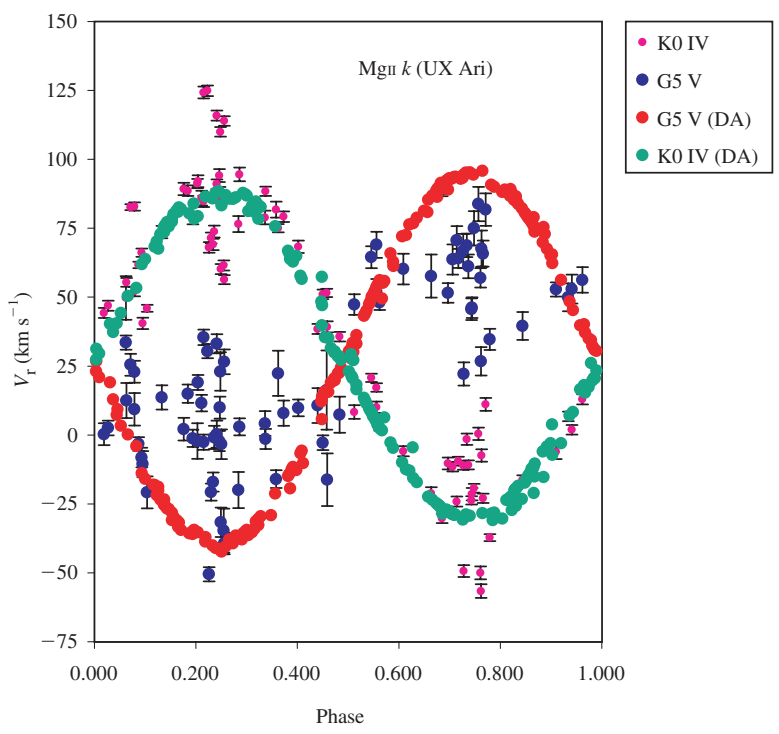

(b)

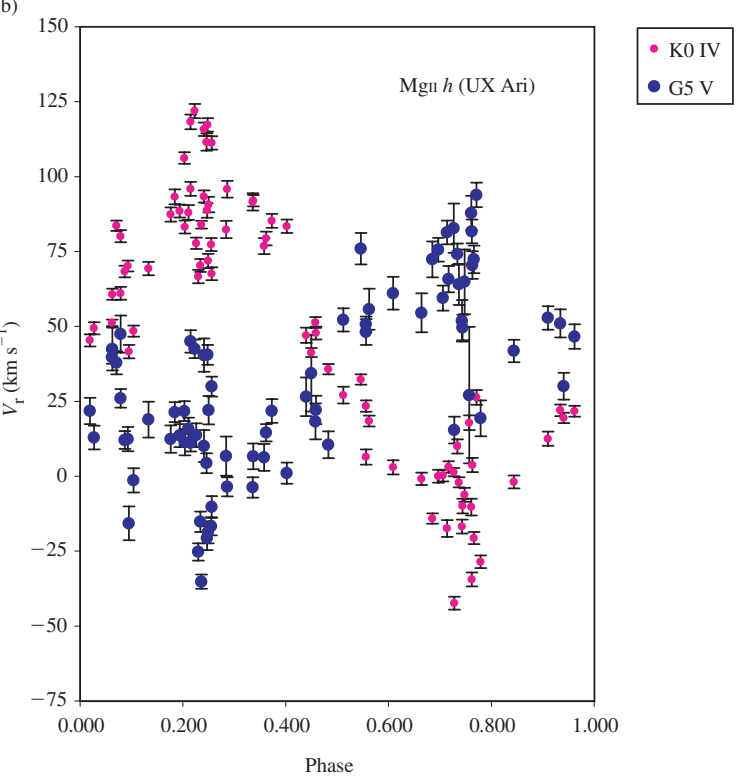

Figure 10 (a) The MgII $k$ radial velocity curves of UX Ari system together with the curves of Duemmler \& Aarum (2001) (with the (DA) legends) which listed in their Table 2, and (b) the MgII $h$ radial velocity curves of UX Ari.

and upper chromosphere) in the years of 1987 (epoch of 1035.732), 1991 (epoch of 1300.757) and 1994 (near the epoch of 1470). However, the periodicity of the flux variation in time was not detected clearly from the 18 years of data. An application of the period search by discrete Fourier transform to OI (middle chromosphere line), CII (upper chromosphere line), Sirv (transition region line) and MgII $k$ emission line fluxes did not give the significant results due to large gaps in the IUE data. On the other hand, the evaluation of the highest flux level of emission lines (occurred at some epochs) showed that the first enhancement was in 1987 (epoch of 1035) occurring 9 years after the first data was obtained in 1978. If the period was 9 years, the next flux enhancement should have been in
1996 (epoch of 1548) instead of 1994 (epoch of 1468). Therefore, the variation with time may be with the periodicity of 7-9 years (which is close to the well known 10-year cycle of the RS Cvn phenomena, see Rodonó 1980). However, this variation was not as clear as that with orbital phase, probably due to the insufficient distribution of the data to determine such a periodicity.

There were two explicit increments around $0.20 P$ and $0.70 P$ in all chromospheric and transition line flux variations depending on the orbital phase. The first flux increment near $0.20 P$ was composed of the data from the spectra taken in 1981 (SWP13162), in 1990 (SWP39460) and in 1994 (SWP51866, SWP51867, SWP51961, SWP51962, SWP52016, SWP52017). The second flux increment near the $0.70 P$ was composed of the data from the spectra taken in 1979 (SWP07267), in 1987 (SWP30026, SWP30027, SWP30028, SWP30029, SWP30030), in 1991 (SWP42416) and in 1994 (SWP52046, SWP52047). The $V$ light-curve amplitudes of UX Ari in these years are $0.16 \mathrm{mag}$ (1981), $0.07 \mathrm{mag}$ (1990), $0.19 \mathrm{mag}$ (1994), $0.04 \mathrm{mag}$ (1979), $0.19 \mathrm{mag}$ (1987) and 0.06 mag (1991) (Raveendran \& Mohin 1995). Therefore, these flux increments did not seem to correlate with the $V$ light-curve amplitudes, but there was a good agreement with the configuration of the component stars near the quadratures. The same situation also appeared in the $\mathrm{MgII} h$ and $k$ emission-line fluxes. By using several optical chromospheric activity indicators, $\mathrm{HeI} \mathrm{D}_{3}, \mathrm{NaI} \mathrm{D}_{1}$, $\mathrm{D}_{2}, \mathrm{H} \alpha$ and CaII IRT lines, Gu et al. (2002) also detected this high activity level of UX Ari around the second quadrature. They suggested that this may originate in the coupling of the chromospheric activity of the secondary and mass-transfer activity of the two components. Another important consideration is that HeII ( $\lambda 1640)$ fluxes may contribute to flux enhancement due to collisional excitation (Athay 1965; Jordan 1975) indicating a temperature of $\sim 8 \times 10^{4} \mathrm{~K}$ and recombination following photoionization by coronal X-rays (Zirin 1976). The contribution of recombination to the HeII flux increases it up to $80 \%$ in the more active region (Rego et al. 1983). Another contributor to HeII is the FeII $\lambda 1640.15$ emission (Jordan 1975; Kohl 1977). Therefore, the HeII ( $\lambda 1640)$ emission feature cannot be considered a pure chromospheric indicator for UX Ari.

Examination of the ultraviolet excess in UX Ari by using the 24 long-wavelength, low-dispersion spectra of UX Ari (see Table 3), and comparison stars $\kappa$ Cet and $\eta$ Cep (see Table 4) in the spectral range between 2100 and $3200 \AA$ showed that there is some ultraviolet excess in UX Ari system which varies from $1 \%$ up to $24 \%$. However, two of these images, LWP31894 and LWP31895, showed no ultraviolet excess for UX Ari system. These 24 spectra were taken in the 1979-1996 period and covered most of the orbital phases. This examination is based on the computation of the theoretical continuum surface fluxes (computed from $\kappa$ Cet and $\eta$ Cep spectra as comparison stars) and the UX Ari continuum surface fluxes in the spectral range mentioned above. Using the same 
comparison stars ( $\kappa$ Cet and $\eta$ Cep) and based on their 1975 observations, Rhombs \& Fix (1977) measured spectrophotometrically the ultraviolet excess in UX Ari in which the cool star contributes $75 \pm 5 \%$ of the total light of the system at $4700 \AA$. Their spectrophotometric observations were carried out at orbital phases 0.715 and 0.791 , and they attributed the wavelength-dependent ultraviolet excess in UX Ari to free-free emission from hot circumstellar gas in the system. There is clearly agreement with the results of Rhombs \& Fix (1977) and this study on the existence of an ultraviolet excess in UX Ari.

The integrated UV continuum fluxes of UX Ari have the lowest flux level of long-wavelength, low-dispersion IUE spectra near the epoch of 634 and these two images (LWR06329L and LWR06329S) were taken about eleven months after the flare event that occurred on 1979 Jan 1. At the time of LWR06329 images (1979 Dec 8), the $V$ light curve of UX Ari had an amplitude about 0.04 mag (Raveendran \& Mohin 1995). The integrated UV continuum fluxes of UX Ari, near the epoch of 1077 (see Table 3), show the variation with the orbital phase, and have the highest flux level of long-wavelength low dispersion IUE spectra. After the flare event appeared in January of 1987, detected from simultaneous IUE and VLA observations (Lang \& Willson 1988), during the time interval from 1987 September 29 to 1987 October 1 (the dates of images from LWP11747 to LWP11770; see Table 3), the $V$ light curve of UX Ari had an amplitude of about 0.19 mag (Raveendran \& Mohin 1995). Near the epoch of 1548 (1996 January), the integrated UV continuum fluxes of UX Ari (see Table 3 ) show the variation with the orbital phase and have lower flux levels than that of the IUE spectra taken in 1987 (near the epoch of 1077). Especially the three images, taken sequentially (LWP31894, LWP31895 and LWP31896) on 1996 Jan 20 show some rise in the UV continuum fluxes near the 0.6 orbital phase. Unfortunately, no photometric, X-ray or radio observation of UX Ari made at this time interval of epoch 1548 has been made to enable comparison with these UV continuum fluxes.

Similar to the emission line fluxes of low dispersion spectra, MgII $h$ and $k$ emission line fluxes of long wavelength high resolution spectra (evaluated from 79 images) have a variation with the orbital phase (Figure 9). There are also some increments of fluxes near 0.20 and 0.70 orbital phases. From the individual MgII emission-line fluxes of the component stars of UX Ari (see Figure 9), it was found that the contributions to the activity of the system of G5 V and K0 IV components are about, on average, $20 \%$ and $80 \%$ respectively, but these ratios varied with time and the orbital phase. However, as mentioned in the introduction of this paper, these contributions are estimated to be about $25 \%$ for G5 V and $75 \%$ for K0 IV from 25 images with IUESIPS reduction, which are not much different from the evaluation given above. Therefore, it can be pointed out that the activity of the system not only comes from the K0 IV component but also comes partially from G5 V component of UX Ari. That means both components have activity phenomena with the most of the contributions to the activity of the system coming from K0 IV. As direct evidence for the activity level of the secondary star of UX Ari, this result confirms the findings and discussions on the secondary component given by Aarum Ulvås \& Engvold (2003), by using CaII K core emission of the secondary.

Although the absorption feature, observed on the peak of the MgII $k$ profiles of the K0 IV component and shifted together with the emission profile as the star revolving on its orbit, was determined on the IUE spectra with IUESIPS reduction (Ekmekçi 1993) this absorption feature was not found to appear on the IUE spectra with NEWSIPS reduction (Figure 6). This discrepancy is likely to originate from the absolute flux errors in the IUESIPS processing summarized by Nichols \& Linsky (1996).

There is a flare event observed on 1979 Jan 1 at 0.062 orbital phase (LWR03344). Also, this flare event appeared in the short wavelength low dispersion IUE image SWP 03766 taken on the same date at 0.068 orbital phase. These two images were also studied by Simon, Linsk \& Schiffer (1980) who gave a plausible explanation for this flare emission, stating that downflowing material from the K0 IV component onto the G5 V star with velocities ranging up to $475 \mathrm{~km} \mathrm{~s}^{-1}$ possibly originates in stellar prominences, or at the base of coronal loops associated with the active regions on the surface of the K0 IV star, or with material streaming between the stars. Their flux estimations for this flare spectrum were $3.8 \times 10^{-11} \mathrm{erg} \mathrm{cm}^{-2} \mathrm{~s}^{-1}$ for MgII $k$ and $3.2 \times 10^{-11} \mathrm{erg} \mathrm{cm}^{-2} \mathrm{~s}^{-1}$ for MgII $h$ emission which are slightly different from the values estimated in this study $\left(4.8 \times 10^{-11} \mathrm{erg} \mathrm{cm}^{-2} \mathrm{~s}^{-1}\right.$ for MgII $k$ and $4.7 \times 10^{-11} \mathrm{erg} \mathrm{cm}^{-2} \mathrm{~s}^{-1}$ for MgII $\left.h\right)$. These differences could have mainly arisen from different reduction procedures (IUESIPS/NEWSIPS). In the Gaussian profile fitting procedure for this flare spectrum (LWR03344) a fourth Gaussian profile (denoted by brown solid line in Figure 7) was added to match the flare event for both $h$ and $k$ emission lines. The flare contribution to the integrated emission line fluxes not only comes from this fourth Gaussian component but also from the G5 V and K0 IV components. The total effect of this flare must be shared, with appropriate amounts, between the G5 V, K0 IV and the conjunction of coronal loops between the component stars of UX Ari system which is located nearer to G5 V star (see Figure 4 of Simon et al. 1980).

Comparison of the radial velocities of MgII $k$ emission line profiles of the components of UX Ari system with those of radial velocities of Duemmler \& Aarum (2001) and of Carlos \& Popper (1971) obtained from the visible spectral range showed some agreements with the data of K0 IV component but the velocities of MgII for G5 V component were, mostly near the quadratures, much different (lower) than the velocities obtained from the visible spectral range (Figure 10a). In addition, the velocities of MgII for G5 V component showed a great scattering by comparison with velocities of MgII for K0 IV component. 
On the account of the effect of the interstellar absorption was removed by the Gaussian profile fitting procedure, this great scattering in the velocities of G5 $\mathrm{V}$ component could likely due to physical interaction between the $\mathrm{K} 0$ IV and G5 V component which is seen actively in UV spectral region. This great scattering and the lower velocities compared to visible data, could make a suggestion for the chromospheric activity via a magnetic dynamo which produced the active region loops. Moreover, the chromospheric instability of G5 V could be due to interaction between the G5 V and K0 IV components via magnetic coronal loops. The mean value of the velocity $\gamma$ of the centre of mass of the system was found to be $34.62 \pm 4.86 \mathrm{~km} \mathrm{~s}^{-1}$ from the MgII $h$ and $k$ radial-velocity curves, which was somewhat greater (about $8 \mathrm{~km} \mathrm{~s}^{-1}$ ) than that of Duemmler \& Aarum (2001) and Carlos \& Popper (1971) $\left(26.5 \mathrm{~km} \mathrm{~s}^{-1}\right)$.

Combining all spectral characteristics of UX Ari supported the model of inhomogeneous gyro-synchrotron emission arising from electrons which have interaction with inhomogeneous magnetic fields (Mutel et al. 1987). As a result of these agreements, the UV emission flux variation with orbital phase in UX Ari might be strongly correlated with the size and configuration of dark spots (see Vogt \& Hatzes 1991 and Aarum \& Engvold 2003) related to the magnetic origin of the activity phenomena. Based on the contribution of G5 V and K0 IV components (on the order of $20 \%$ and $80 \%$, respectively) to the MgII activity of the system, it would be suggested that it is better to take into consideration the spot distribution not only on the surface of K0 IV but also on the surface of the G5 V component of UX Ari. However, some constraints on the secondary component were given by Aarum Ulvås \& Engvold (2003).

\section{Acknowledgments}

I would like to thank Randy Thompson for his kind help in converting NEWSIPS files to ASCII formats by using IDL on the IUE account. I also thank Mesut Yilmaz and Tolga Colak for their assistance in compiling the manuscript with latex. And finally, I would like to thank the referee for his/her cautions on some points to improve the result of this study. This research has made use of the Simbad database, operated at CDS, Strasbourg, France, and of NASA's Astrophysics Data System Bibliographic Services.

\section{References}

Aarum Ulvås, V. \& Engvold, O., 2003, A\&A, 402, 1043

Athay, R. G., 1965, ApJ, 142, 755

Bevington, P. R., 1969, Data Reduction and Error Analysis for The Physical Sciences (New York: McGraw-Hill), 237

Blackwell, D. E. \& Lynas-Gray, A. E., 1994, A\&A, 282, 899

Carlos, R. C. \& Popper, D. M., 1971, PASP, 83, 504

Duemmler, R. \& Aarum, V., 2001, A\&A, 370, 974

Ekmekçi, F., 1993, PhD Thesis, Ankara University Graduate School of Natural and Applied Sciences

Gray, D. F., 1992, The Observations and Analysis of Stellar Photospheres (Second Edition; New York: Cambridge University Press), 340

Gu, S.-h., Tan, H.-s., Shan, H.-g. \& Zhang, F.-h., 2002, A\&A, 388,889

Huenemoerder, D. P., Buzasi, D. L. \& Ramsey, L. W., 1989, AJ, 98, 1398

Jordan, C., 1975, MNRAS, 170, 429

Kohl, J. L., 1977, ApJ, 211, 958

Lang, K. R. \& Willson, R. F., 1988, ApJ, 328, 610

Montes, D., Fernández-Figueroa, M. J., De Castro, E. \& Cornide, M., 1995a, A\&AS, 109, 135

Montes, D., Fernández-Figueroa, M. J., De Castro, E. \& Cornide, M., 1995b, A\&A, 294, 165

Mutel, R. L., Morris, D. H., Doiron, D. J. \& Lestrade, J. F., 1987, AJ, 93, 1220

Nichols, J. S. \& Linsky, J. L., 1996, AJ, 111, 517

Perryman, M. A. C. et al., 1997, A\&A, 323L, 49

Raveendran, A. V. \& Mohin, S., 1995, A\&A, 301, 788

Rego, M., Gonzalez-Riestra, R. \& Fernández-Figueroa, M. J., 1983, A\&A, 119, 227

Rhombs, C. G. \& Fix, J. D., 1977, ApJ, 216, 503

Rodonó, M., 1980, MmSAI, 51, 623

Simon, T., Linsky, J. L. \& Schiffer, F. H., 1980, ApJ, 239, 911

Soubiran, C., Bienaymé, O., Mishenina, T. V. \& Kovtyukh, V. V., 2008, A\&A, 480, 91

Strassmeier, K. G. et al., 1988, A\&AS, 72, 291

Strassmeir, K. G., Hall, D. S., Fekel, F. C. \& Scheck, M., 1993, A\&AS, 100, 173

Vogt, S. S. \& Hatzes, A. P., 1991, IAUC, 130, 297

Zirin, H., 1976, ApJ, 208, 414 